\title{
Miniband Structure of Quantum Dots based on GaN/AlN Nanowire Arrays
}

\author{
Oui Chan Jung, Hyung Uk Cho, and Jong Chang Yi* \\ Electronic Eng. Department, Hongik University, Sangsu 72-1, Mapo, Seoul 121-791, Republic of Korea
}

(Received April 16, 2008 : revised May 23, 2008 : accepted May 27, 2008)

\begin{abstract}
The miniband structure of a quantum dot lattice based on GaN/AlN nanowire arrays has been investigated using the finite element method and Floquet theorem. The quantum dot modes and the quantum wire modes in the nanowire arrays were graphically verified. The optimum geometries of GaN/AlN quantum wire arrays were investigated by using a correlation between the width of nanowires and the separation of the minibandgap which is to be larger than the thermal energy at room temperature.
\end{abstract}

Keyword: Quantum dot, Nanowire array, Miniband, Super lattice, Finite-element method

OCIS codes : (000.1600) Classical and quantum physics; (270.0270) Quantum optics; (020.5580) Quantum electrodynamics; (270.5580) Quantum electrodynamics.

\section{INTRODUCTION}

Uniform quantum dot arrays have significant potential advantages due to the localized density of states; they are expected to give lower power consumption and higher differential gain [1] once applied to optoelectronic devices. Although quantum dot structures have been extensively investigated, the fabrication of uniform three-dimensional nanostructures for sufficient quantum size effect remains an ongoing research topic. Recently, quantum dot structures based on the edge-defined quantum structure $[2,3]$ have been reported and investigated. The crossing points between top and bottom quantum wires from the edgedefined quantum structure form two dimensional superlattice quantum dots. Although more advanced fabrication technologies are required to put the quantum dot array laser diodes to practical use, the primary advantage of such structure is the enhancement of the uniformity and density of the quantum dots. The uniformity and the density of the nanowire structures are governed by the multiple quantum well thickness which usually ranges between a few $\mathrm{nm}$ to several $\mathrm{nm}$.

In quantum dot arrays, the electron energy states form minibands due to the coupling between dots $[3,4]$. However, the electrons usually have an energy uncertainty of the thermal energy, $k T$, where $k$ is the

\footnotetext{
*Corresponding author: wave@hongik.ac.kr
}

Boltzmann constant and $T$ is the temperature. Due to this electron energy uncertainty, the miniband gap needs to be larger than $2 \mathrm{kT}$ to observe the quantum size effect due to the localization of the density of states at room temperature. In this work, the miniband structures of the quantum dot arrays based on crossed nanowires have been analyzed by using the finite- element method with periodic boundary conditions [4-6]. The formation of quantum dot mode is graphically verified by investigating the three dimensional electron mode profiles. Their miniband properties such as the $E-k$ diagram and miniband gap between quantum dot and quantum wire modes were also quantitatively analyzed.

\section{MINIBAND CHARACTERIZATION}

Figure 1 shows the schematic diagram of the quantum wire junction. The base material is intrinsic AlN and the material of the nanowires is intrinsic GaN. The widths of rectangular wire wells and barriers are both $3 \mathrm{~nm}$. The quantum wire junctions are formed at the crossing points between top and bottom nanowires. The number of nanowire is 50-100. Thus, one can see that the quantum wire junctions repeat 50-100 times along the $x$ - and $y$-directions. Therefore, one can safely assume that the quantum wire junctions are periodic 


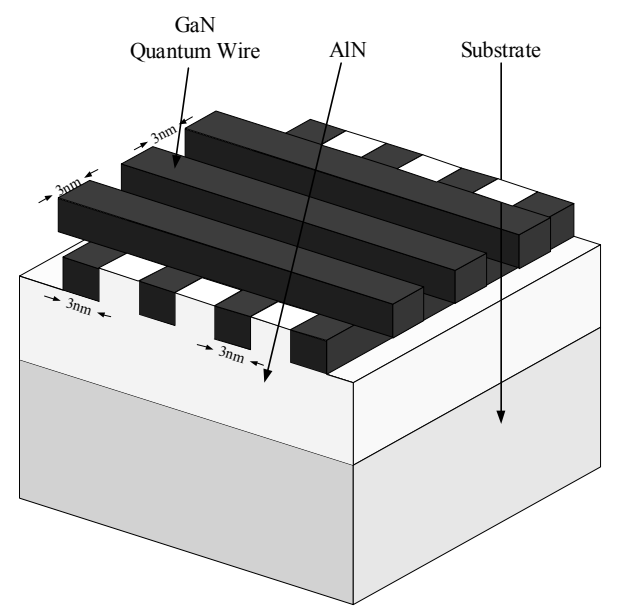

FIG. 1. The schematic diagram of the quantum wire junction

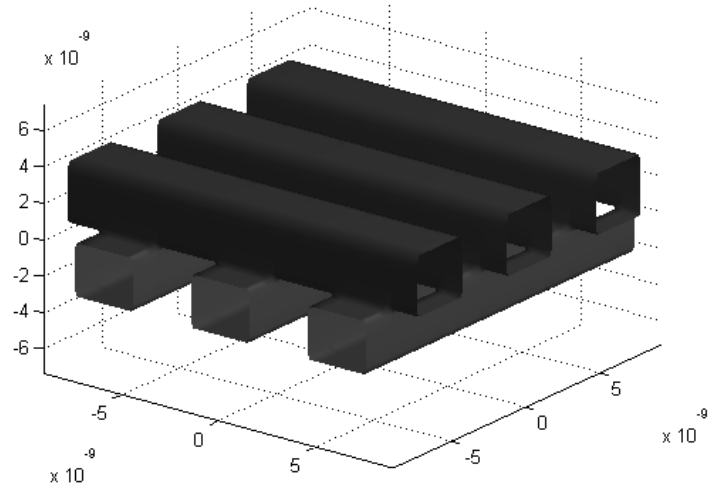

FIG. 2. Rectangular quantum wire structures with a period of $6 \mathrm{~nm}$ along the $x$ - and $y$-directions.

along both the $x$ - and $y$-directions with a lattice constant $\mathrm{L}_{\mathrm{x}}$ and $\mathrm{L}_{\mathrm{y}}$ of $6 \mathrm{~nm}$ as shown in Fig. 2. The Floquet theorem states that the electron wave functions at the boundary of periodic structure can be expressed as shown below:

$$
\begin{aligned}
& \Psi\left(L_{x}, y, z\right)=e^{j \phi_{x}} \Psi(0, y, z) \\
& \Psi\left(x, L_{y}, z\right)=e^{j \phi_{y}} \Psi(x, 0, z)
\end{aligned}
$$

Here $\phi_{\mathrm{x}}$ and $\phi_{\mathrm{y}}$ denote the phase change per period varying from $-\pi$ to $\pi$.

Figure 3 shows the wave functions for a 3 by 3 lattice along the $x$ - and $y$-directions. Fig. 3(a) shows the electron density profile for the fundamental mode which clearly shows a 3 dimensional quantum confinement. Fig. 3(b)-(e) shows the electron density profiles for the higher modes. Fig. 3 (b) clearly shows that the second mode is a quantum wire-like mode which has a 2 dimensional confinement only. From Fig 3 (a) and (b), one can see that the fundamental mode is a true quantum

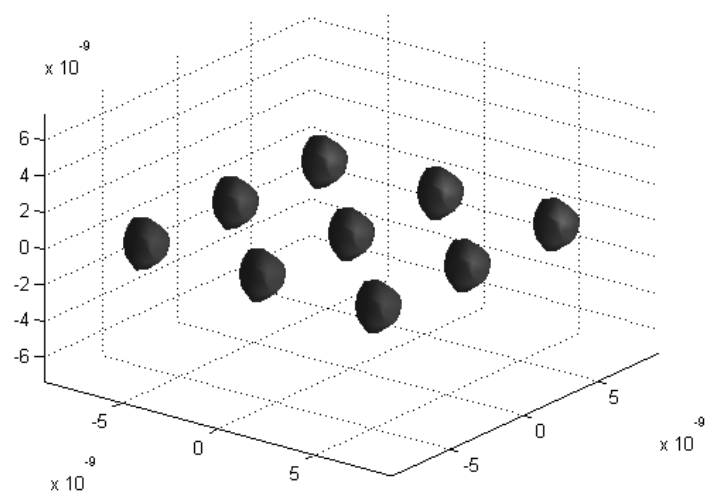

FIG. 3. (a) The electron density profile for the fundamental mode when $\phi_{\mathrm{x}}=\phi_{\mathrm{y}}=0$.

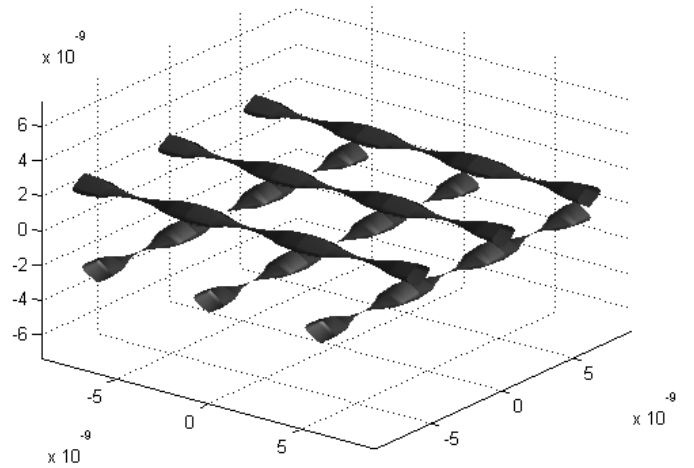

FIG. 3. (b) The electron density profile for the second mode when $\phi_{\mathrm{x}}=\phi_{\mathrm{y}}=0$.

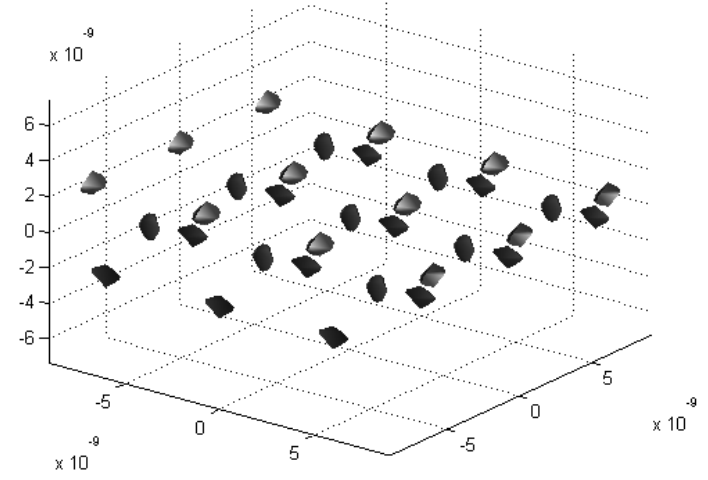

FIG. 3. (c) The electron density profile for the third mode when $\phi_{\mathrm{x}}=\phi_{\mathrm{y}}=0$.

dot mode and the second mode is a quantum wire-like mode. One thing to note here is that the forth and fifth modes are the degenerate states due to the rotational symmetry of the structure.

Figure 4 shows the complete $E-k$ diagram when the width and the distance of nanowires were $3 \mathrm{~nm}$. The vertical axis is for electron quantized energy states above the conduction band of the quantum well material in $e V$ unit. The horizontal axis is for the phase changes 


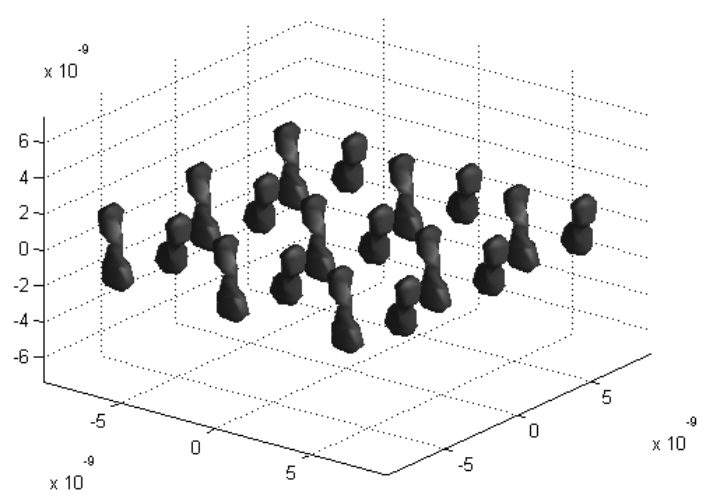

FIG. 3. (d) The electron density profile for the fourth mode when $\phi_{\mathrm{x}}=\phi_{\mathrm{y}}=0$.

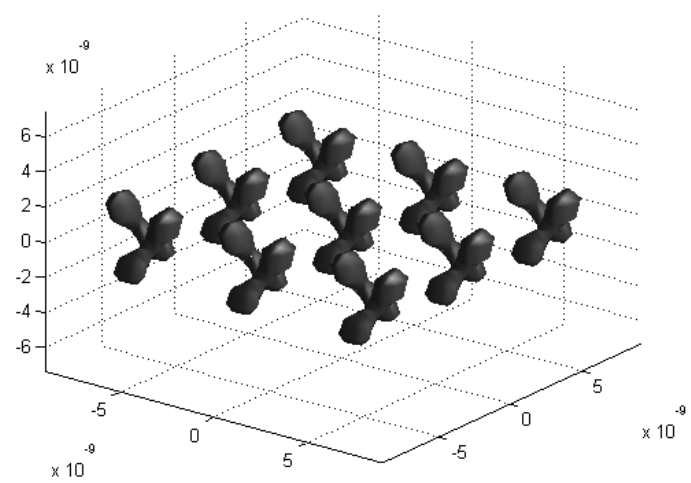

FIG. 3. (e) The electron density profile for the fifth mode when $\phi_{\mathrm{x}}=\phi_{\mathrm{y}}=0$.

along $\mathrm{x}$ - and $\mathrm{y}$-axis divided by $\pi$. From -1 to $0, \phi_{\mathrm{x}}$ and $\phi_{\mathrm{y}}$ vary simultaneously from $\pi$ to 0 ; from 0 to $1, \phi_{\mathrm{x}}$ varies from 0 to $\pi$ while maintaining $\phi_{\mathrm{y}}=0$; from 1 to $2, \phi_{\mathrm{y}}$ varies from 0 to $\pi$ with $\phi_{\mathrm{x}}=\pi$. The miniband gap is defined as the energy gap between the maximum value of first quantized energy (quantum dot mode) and the minimum value of second quantized energy (quantum wire mode). In Figure 4, it is $0.0567 \mathrm{eV}$. This miniband gap is twice as large as the thermal energy $k T$ at room temperature.

Figure 5 and 6 show the miniband structures for the same nanowire structures but with different wire sizes. Fig. 5 shows the complete $E-k$ diagram when the width and the separation of the nanowires are $4 \mathrm{~nm}$. In this case, the miniband gap is $0.0385 \mathrm{eV}$. Figure 6 shows the $E-k$ diagram when the wire width and separation are $2 \mathrm{~nm}$ only. In this case, the miniband gap is 0.1075 eV. Figure 7 shows the variation of the minibandgap energy versus the nanowire width. From Fig. 7, one can see that the minibandgap becomes smaller than 2 $k T$ when the wire width becomes larger than $4 \mathrm{~nm}$. Therefore, one can conclude that an optimum quantum dot structure on the crossed nanowires should have lateral dimensions smaller than $3 \mathrm{~nm}$ to obtain a

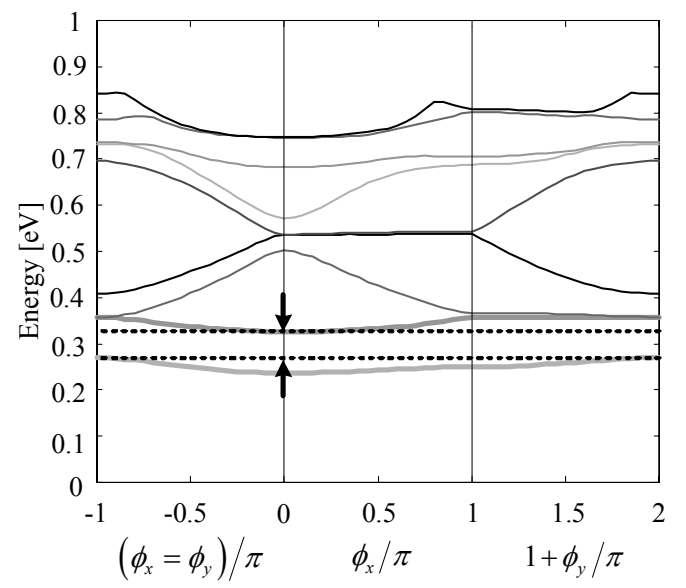

FIG. 4. The $E-k$ diagram of quantum dot arrays on quantum wire junctions when the width and the distance of the nanowires $=3 \mathrm{~nm}$.

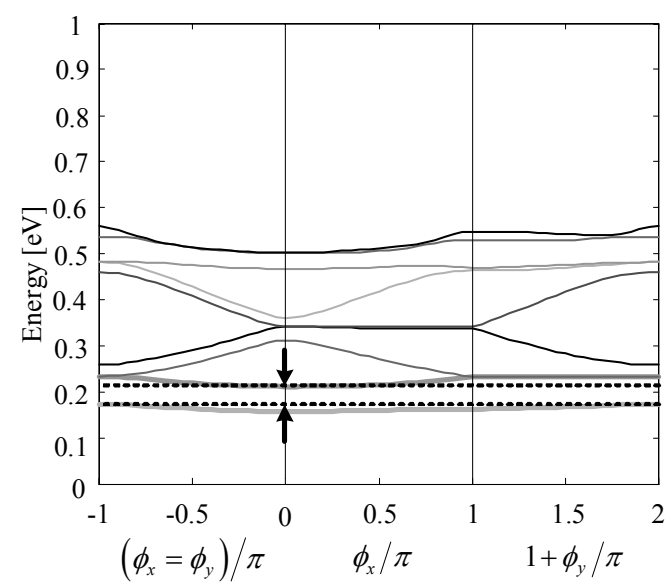

FIG. 5. The $E-k$ diagram of quantum dot arrays on quantum wire junctions when the width and the distance of the nanowires $=4 \mathrm{~nm}$.

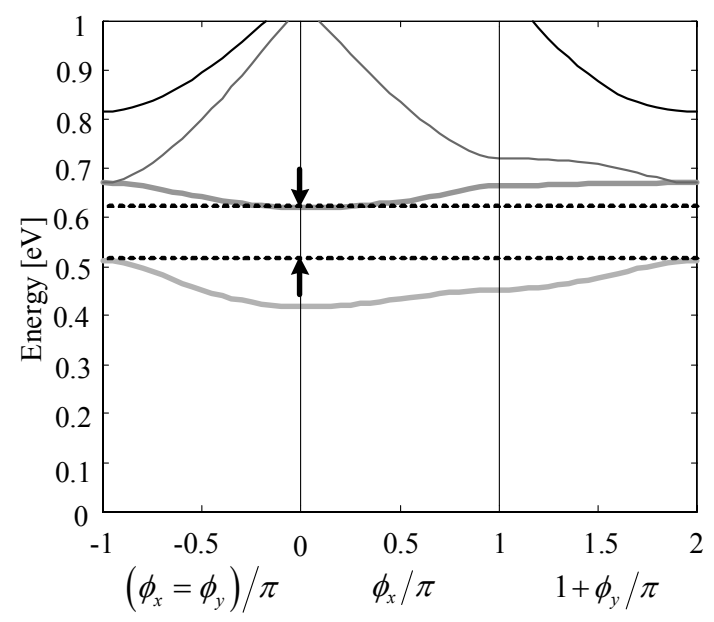

FIG. 6. The $E-k$ diagram of quantum dot arrays on quantum wire junctions when the width and the distance of the nanowires $=2 \mathrm{~nm}$. 


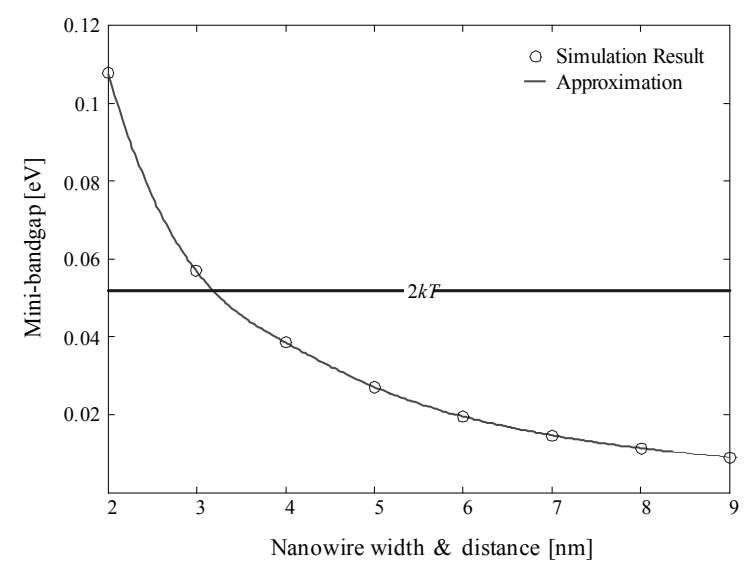

FIG. 7. The variation of the minibandgap energy versus the nanowire width and the separation.

miniband gap larger than $2 k T$ at room temperature.

\section{CONCLUSION}

Uniform quantum dot arrays based on the edge-defined quantum wires in GaN/AlN were analyzed. The quantum dot mode and the quantum wire mode were graphically verified, and the optimum periodic structure for a miniband gap to be larger than $2 k T$ at room temperature was estimated. Primary advantage of such quantum dot arrays is the enhancement of the uniformity and density of the quantum dots since the lateral dimension is defined by the MQW thickness. These characteristics will lead to the reduced power consumption and enhanced device performances.

\section{ACKNOWLEDGEMENT}

This work was supported by the Korea Science and Engineering Foundation (KOSEF) grant funded by the Korea government (MOST) under R01-2007-000-20048-0.

\section{REFERENCES}

[1] H. Sasaki, "Quantum wires, quantum boxes and related structures: Physics, device potentials and structural requirements," Surface Science., vol. 267, pp. 623-629, 1992.

[2] N. A. Melosh, A. Boukai, F. Amsinck, A. Badolato, P.M. Petroff, and J.R. Heath, "Ultrahigh-density nanowire lattices and circuits," Science, vol. 300, pp. 112-115, 2003.

[3] J. C. Yi, "Miniband properties of superlattice quantum dot arrays fabricated by the edge-defined nanowires," Microelectronics Journal, vol. 39, no. 3, pp. 369-374, 2008.

[4] J. C. Yi, N. Dagli, and L. A. Coldren, "Investigation of titled superlattices for quantum wire laser applications," Appl. Phys. Lett, vol. 59, pp. 3015-3017, 1991.

[5] J. C. Yi and J. B. Ji, "Band Structure Analysis of Strained Quantum Wire Array," Journal of Optical Society of Korea, vol.7, no.1, pp. 7-12, 2003.

[6] J. C .Yi, N. Dagli, "Finite-element analysis of valance band structure and optical properties of quantum-wire arrays on vicinal substrates," IEEE J. Quantum Electron, vol. 31, pp. 208-218, 1995. 